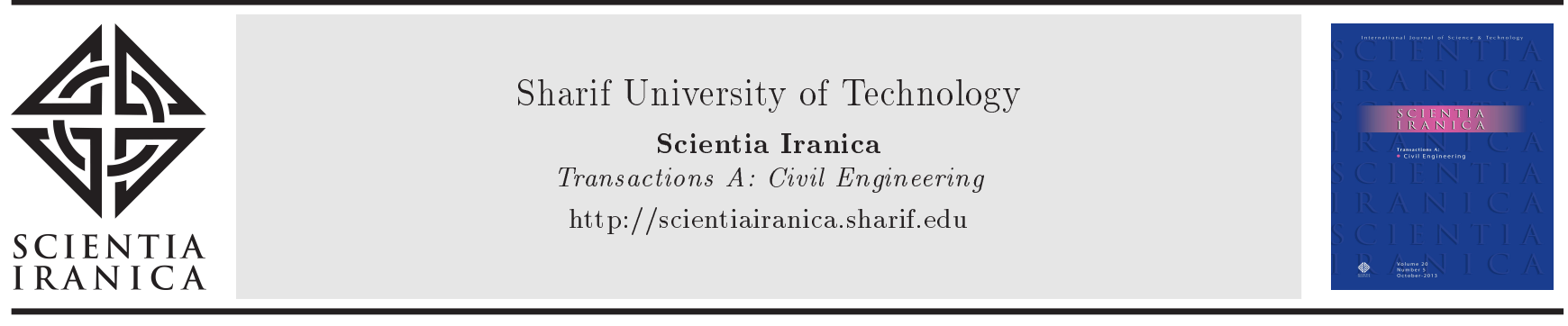

\title{
Dynamic analysis of concrete gravity dam-reservoir systems by a wavenumber approach for the general reservoir base condition
}

\author{
M. Jafari* and V. Lotfi \\ Department of Civil and Environmental Engineering, Amirkabir University of Technology, Tehran, Iran.
}

Received 19 October 2016; received in revised form 18 February 2017; accepted 3 July 2017

KEYWORDS
Frequency domain
analysis;
Concrete gravity
dams;
Wavenumber
approach;
Reservoir bottom
absorption;
Truncation boundary.

\section{KEYWORDS}

analysis;

Concrete gravity

dams;

Wavenumber

approach;

absorption;

Truncation boundary.

\begin{abstract}
Different approaches are utilized for dynamic analysis of concrete gravity dam-reservoir systems. The rigorous approach to solving this problem employs a twodimensional semi-infinite fluid element (i.e., hyper-element). Recently, a technique has been proposed to perform the dynamic analysis of dam-reservoir systems in the pure finiteelement programming context, referred to as the wavenumber approach. Of course, certain limitations have been imposed on the reservoir base condition in the initial form of this technique to simplify the problem. However, this is presently discussed for the general reservoir base condition, contrary to the previous study which was merely limited to the fully reflective reservoir base case. In this technique, the wavenumber condition is imposed on the truncation boundary or the upstream face of the near-field water domain. The method is initially described. Subsequently, the response of an idealized triangular damreservoir system is obtained by this approach, and the results are compared against those of the exact response. Based on this investigation, it is concluded that this approach can be envisaged as a great substitute for the rigorous type of analysis under the general reservoir base condition.
\end{abstract}

(C) 2018 Sharif University of Technology. All rights reserved.

\section{Introduction}

Dynamic analysis of concrete gravity dam-reservoir systems can be carried out rigorously by FE-(FE-HE) method in the frequency domain. This means that the dam is discretized by plane solid finite elements, while, the reservoir is divided into two parts: a near-field region (usually an irregular shape) in the vicinity of the dam and a far-field part (assuming uniform depth) which extends to infinity in the upstream direction.

\footnotetext{
*. Corresponding author.

E-mail addresses: mehranj@aut.ac.ir (M. Jafari);

vahlotfi@aut.ac.ir (V. Lotfi)
}

The former region is discretized by plane fluid finite elements and the latter part is modelled by a twodimensional fluid hyper-element [1-3]. It is well-known that employing fluid hyper-elements would lead to the exact solution of the problem. However, it is formulated in the frequency domain and its application in this field has led to many special purpose programs, which are demanding from the programming point of view.

On the other hand, engineers have often managed to solve this problem in the context of pure finiteelement programming (FE-FE method of analysis). In this approach, an often simplified condition is imposed on the truncation boundary or the upstream face of the near-field water domain. Thus, the fluid hyperelement is actually excluded from the model. Some of 
these widely used simplified conditions [4,5] may result in significant errors if the reservoir length is small, and it might lead to high computational cost if the truncation boundary is located at far distances. The main advantage of these conditions is that it can be readily used for time domain analysis. Thus, they are vastly employed in nonlinear seismic analysis of concrete dams.

Of course, many studies in the last three decades have developed more accurate absorbing boundary conditions to apply to similar fluid-structure or soilstructure interaction problems. Perfectly matched layers [6-11] and high-order non-reflecting boundary condition [12-18] are among the two main popular groups of methods which researchers have applied in their attempts. It is emphasized that these techniques have become very popular in recent years because they could be applied to the time and frequency domains. However, it should be realized that they are not very attractive in the frequency domain. This is well understood that they are not very simple to use and, more importantly, they are compared with the hyperelement alternative which produces exact results, no matter how small the near-field reservoir length is.

In the present study, the FE-FE analysis technique is employed as the basis of the proposed method for dynamic analysis of the concrete dam-reservoir system in the frequency domain, which is referred to as the wavenumber approach. The method simply applies an absorbing boundary condition on the truncation boundary which is referred to as the wavenumber condition. It is as simple as employing the Sommerfeld or Sharan condition on the truncation boundary. It should be mentioned that this is an extension and generalization of a previous study which was merely limited to the fully reflective reservoir base case $[19,20]$. This is presently discussed for the general reservoir base condition.

In the following sections of the article, the method of analysis is initially explained. Subsequently, the response of an idealized triangular dam is studied due to the horizontal ground motion for several alternatives employed as an absorbing boundary condition. In each case and regarding the proposed option, the results are compared against the exact solution. The results are provided for the medium- and short-sized reservoir lengths. Moreover, both fully reflective and absorptive reservoir base conditions are studied.

\section{Method of analysis}

As mentioned, the analysis technique utilized in this study is based on the FE-FE method, which is applicable to a general concrete gravity dam-reservoir system. The coupled equations can be obtained by considering each region separately and, then, combining the resulting equations.

\subsection{Dam body}

Concentrating on the structural part, the dynamic behavior of the dam is described by the well-known equation of structural dynamics [21]:

$$
\mathbf{M} \ddot{\mathbf{r}}+\mathbf{C} \dot{\mathbf{r}}+\mathbf{K r} \mathbf{r}=-\mathbf{M} \mathbf{J} \mathbf{a}_{g}+\mathbf{B}^{T} \mathbf{P},
$$

where $\mathbf{M}, \mathbf{C}$, and $\mathbf{K}$ in this relation represent the mass, damping, and stiffness matrices of the dam body. Moreover, $\mathbf{r}$ is the vector of nodal relative displacements, $\mathbf{J}$ is a matrix with each of two rows being equal to a $2 \times 2$ identity matrix (its columns correspond to a unit of horizontal and vertical rigid body motions), and $\mathbf{a}_{g}$ denotes the vector of ground accelerations. Furthermore, $\mathbf{B}$ is a matrix which relates vectors of hydrodynamic pressures (i.e., $\mathbf{P}$ ) and their equivalent nodal forces.

Let us now consider harmonic excitation with frequency $\omega$ and limit the present study to the horizontal ground motion only. It is well known that the response will also behave harmonically (i.e., $\mathbf{r}(t)=\mathbf{r}(\omega) e^{i \omega t}$ ). Thus, Eq. (1) can be expressed as follows:

$$
\left(-\omega^{2} \mathbf{M}+(1+2 \beta i) \mathbf{K}\right) \mathbf{r}=-\mathbf{M} \mathbf{J} \mathbf{a}_{g}^{h}+\mathbf{B}^{T} \mathbf{P} .
$$

In this relation, it is assumed that the damping matrix of the dam is of hysteretic type. This means that:

$$
\mathbf{C}=(2 \beta / \omega) \mathbf{K}
$$

Moreover, it should be emphasized that superscript $h$ on the acceleration vector refers to the horizontal type of excitation. That is:

$$
\mathbf{a}_{g}^{h}=\left(\begin{array}{c}
a_{g}^{x} \\
0
\end{array}\right) \text {. }
$$

\subsection{Water domain}

Assuming that water is linearly compressible with intentional disregard for its viscosity, its small irrotational motion (Figure 1 ) is governed by the wave equation $[22,23]$ :

$$
\frac{\partial^{2} p}{\partial x^{2}}+\frac{\partial^{2} p}{\partial y^{2}}-\frac{1}{c^{2}} \ddot{p}=0 \quad \text { in } \Omega \text { and } D
$$

where $p$ is the hydrodynamic pressure, and $c$ is the pressure wave velocity in water. The boundary conditions for reservoir's surface and bottom are as follows:

$$
p=0 \text { on the water surface, }
$$

$$
\frac{\partial p}{\partial n}=-\rho a_{\mathrm{g}}^{n}-q \dot{p} \quad \text { at the reservoir's bottom. }
$$

Herein, $\rho$ is the water density, and $n$ denotes the outward (with respect to fluid region) perpendicular 
direction at the reservoir's bottom. Moreover, the admittance or damping coefficient $q$ utilized in the above equation may correspond to a more meaningful wave reflection coefficient $\alpha$ [24]:

$$
\alpha=\frac{1-q c}{1+q c},
$$

which is defined as the ratio of the amplitude of the reflected hydrodynamic pressure wave to the amplitude of a vertically propagating pressure wave incident on the reservoir bottom. For a fully reflective reservoir's bottom condition, $\alpha$ is equal to 1 which leads to $q=0$.

One can apply the weighted residual approach to obtaining the finite-element equation of the fluid domain, which may be written as follows:

$$
\mathbf{G}^{e} \ddot{\mathbf{P}}^{e}+\mathbf{H}^{e} \mathbf{P}^{e}=\mathbf{R}^{e}
$$

with the following definitions:

$$
\begin{aligned}
\mathbf{G}^{e} & =\frac{1}{\rho c^{2}} \int_{\Omega_{e}} \mathbf{N ~}^{T} d \Omega, \\
\mathbf{H}^{e} & =\frac{1}{\rho} \int_{\Omega_{e}}\left(\mathbf{N}_{x} \mathbf{N}_{x}^{T}+\mathbf{N}_{y} \mathbf{N}_{y}^{T}\right) \mathrm{d} \Omega, \\
\mathbf{R}^{e} & =\frac{1}{\rho} \oint_{\Gamma^{e}} \mathbf{N}\left(\partial_{n} p\right) d \Gamma^{e},
\end{aligned}
$$

where $\mathbf{N}$ is the vector of element's shape functions, and $\mathbf{N}_{x}, \mathbf{N}_{y}$ denote its partial derivatives with respect to $x$, $y$, respectively. It is also worthwhile to emphasize that superscript $\left({ }^{e}\right)$ states that these matrices are related to the element level. Directional derivative $\partial_{n} p$ in Eq. (9c) can take three forms on different boundaries of the reservoir (Figure 1):

- On the upstream boundary of the reservoir $\left(\Gamma_{\mathrm{I}}\right)$ : One can apply different absorbing boundary conditions which will be discussed in the next section.



Figure 1. Schematic view of a typical dam-reservoir system. The near-field reservoir domain, $\Omega$, truncation boundary, $\Gamma_{1}$, and far-field region, $D$ (excluded in the FE-FE type of analysis).
- At the bottom of the reservoir $\left(\Gamma_{\text {II }}\right)$, one can utilize Eq. (6b) as mentioned previously:

$$
\partial_{n} p=-\rho a_{\mathrm{g}}^{n}-q \dot{p} .
$$

- On the dam-reservoir interface $\left(\Gamma_{\mathrm{III}}\right)$ :

$$
\partial_{n} p=-\rho \ddot{u}_{n},
$$

where $\ddot{u}_{n}$ is the total acceleration of fluid particles normal to the dam-reservoir interface. It is also noted that there must be acceleration compatibility between the fluid and solid particles in that direction.

In general, an element may have all the three abovementioned boundary condition types. Thus, one can write $\mathbf{R}^{e}$ vector as follows:

$$
\mathbf{R}^{e}=\mathbf{R}_{\mathrm{I}}^{e}+\mathbf{R}_{\mathrm{II}}^{e}+\mathbf{R}_{\mathrm{III}}^{e}
$$

Of course, it is possible that some of these boundary condition types are not applied to a certain element, which that part should be eliminated for that specific element. It is easily shown that one would obtain the following relations by utilizing Eqs. (10a) and (10b) in Eq. (9c), respectively:

$$
\begin{aligned}
& \mathbf{R}_{\mathrm{II}}^{e}=-\mathbf{B}_{\mathrm{II}}^{e} \mathbf{J}^{e} \mathbf{a}_{g}^{h}-q \mathbf{L}_{\mathrm{II}}^{e} \dot{\mathbf{P}}^{e}, \\
& \mathbf{R}_{\mathrm{III}}^{e}=-\mathbf{B}_{\mathrm{III}}^{e}\left(\ddot{\mathbf{r}}^{e}+\mathbf{J}^{e} \mathbf{a}_{g}^{h}\right),
\end{aligned}
$$

with the following definitions:

$$
\begin{aligned}
\mathbf{B}_{i}^{e} & =\int_{\Gamma_{i}^{e}} \mathbf{N ~ n}^{T} \mathbf{N}_{s}^{T} \mathrm{~d} \Gamma^{e} ; \quad i \in\{\mathrm{II}, \mathrm{III}\} \\
\mathbf{L}_{\mathrm{II}}^{e} & =\frac{1}{\rho} \int_{\Gamma_{\mathrm{II}}^{e}} \mathbf{N ~ N}^{T} \mathrm{~d} \Gamma^{e} .
\end{aligned}
$$

Herein, $\mathbf{n}$ represents a unit outward normal vector. Moreover, $\mathbf{N}_{s}$ is the matrix of adjacent solid element shape functions utilized to interpolate accelerations in horizontal and vertical directions. It is worthwhile to mention that, from a practical standpoint, the values of non-zero solid and fluid shape functions are essentially equal on the common fluid-solid interface. Substituting Eqs. (12a) and (12b) into Eq. (11) will result in:

$$
\mathbf{R}^{e}=\mathbf{R}_{\mathrm{I}}^{e}-q \mathbf{L}_{\mathrm{II}}^{e} \dot{\mathbf{P}}^{e}-\mathbf{B}^{e} \ddot{\mathbf{r}}^{e}-\mathbf{B}^{e} \mathbf{J}^{e} \mathbf{a}_{g}^{h}
$$

with the following definition:

$$
\mathbf{B}^{e}=\mathbf{B}_{\mathrm{II}}^{e}+\mathbf{B}_{\mathrm{III}}^{e} .
$$

It should be also noted that the relative acceleration at boundary $\Gamma_{\text {II }}$ is identically equal to zero. Subsequently, Eq. (14) can be substituted into Eq. (8) which yields:

$$
\mathbf{G}^{e} \ddot{\mathbf{P}}^{e}+q \mathbf{L}_{\mathrm{II}}^{e} \dot{\mathbf{P}}^{e}+\mathbf{H}^{e} \mathbf{P}^{e}=\mathbf{R}_{\mathrm{I}}^{e}(t)-\mathbf{B}^{e} \ddot{\mathbf{r}}^{e}-\mathbf{B}^{e} \mathbf{J}^{e} \mathbf{a}_{g}^{h}
$$


The equivalent form of this equation in the frequency domain would be:

$$
\begin{aligned}
-\omega^{2} \mathbf{G}^{e} \mathbf{P}^{e} & +i \omega q \mathbf{L}_{\mathrm{II}}^{e} \mathbf{P}^{e}+\mathbf{H}^{e} \mathbf{P}^{e}=\mathbf{R}_{\mathrm{I}}^{e}(\omega) \\
& +\omega^{2} \mathbf{B}^{e} \mathbf{r}^{e}-\mathbf{B}^{e} \mathbf{J}^{e} \mathbf{a}_{g}^{h} .
\end{aligned}
$$

Herein, $\mathbf{L}_{\mathrm{II}}^{e}$ is a matrix that corresponds to the absorption of energy at reservoir's bed. By assembling the element equations and imposing the free surface condition in Eq. (6a), one would obtain the overall FE equation of the fluid domain:

$$
-\omega^{2} \mathbf{G} \mathbf{P}+i \omega q \mathbf{L}_{\mathrm{II}} \mathbf{P}+\mathbf{H} \mathbf{P}=\mathbf{R}_{\mathrm{I}}+\omega^{2} \mathbf{B} \mathbf{r}-\mathbf{B} \mathbf{J} \mathbf{a}_{g}^{h} .
$$

In this equation, $\mathbf{R}_{\mathrm{I}}$ is obtained by assembling the boundary integrals of Eq. (9c) on $\Gamma_{I}$.

\subsection{Dam-reservoir system}

The necessary equations for both dam and reservoir domains were developed in the previous sections. Thus, combining the main relations (18) and (2) would result in the FE equations of the coupled dam-reservoir system in its initial form for the frequency domain:

$$
\begin{gathered}
{\left[\begin{array}{cc}
-\omega^{2} \mathbf{M}+(1+2 \beta i) \mathbf{K} & -\mathbf{B}^{T} \\
-\omega^{2} \mathbf{B} & \left(-\omega^{2} \mathbf{G}+i \omega q \mathbf{L}_{\mathrm{II}}+\mathbf{H}\right)
\end{array}\right]} \\
\left\{\begin{array}{l}
\mathbf{r} \\
\mathbf{P}
\end{array}\right\}=\left\{\begin{array}{c}
-\mathbf{M} \mathbf{J} \mathbf{a}_{g}^{h} \\
\left(-\mathbf{B ~ J ~} \mathbf{a}_{g}^{h}+\mathbf{R}_{\mathbf{I}}\right)
\end{array}\right\}
\end{gathered}
$$

It is noted from the above equation that vector $\mathbf{R}_{\mathrm{I}}$ still needs to be defined by some appropriate conditions. This is related to truncated boundary $\Gamma_{I}$ which will be discussed below.

\subsection{Modification due to truncation boundary contribution}

The effect of truncation boundary will be treated in this section. For this purpose, let us now assume that this boundary (i.e., $\Gamma_{I}$ ) is vertical (i.e., along $y$ direction) and consider a harmonic plane wave with a unit amplitude and frequency $\omega$ propagating along a direction which makes an angle $\theta$ with a negative $x$ direction. This may be written in many different forms such as:

$$
\begin{aligned}
& p=e^{i\left(k^{\prime} x+\lambda y+\omega t\right)}, \\
& p=e^{(i \omega / c)[(\cos \theta) x+(\sin \theta) y+c t]},
\end{aligned}
$$

with the following relations being valid:

$$
\begin{aligned}
& k^{\prime}=\frac{\omega}{c} \cos \theta, \\
& \lambda=\frac{\omega}{c} \sin \theta, \\
& k^{\prime 2}+\lambda^{2}=\frac{\omega^{2}}{c^{2}} .
\end{aligned}
$$

It is easily verifiable that the following condition is appropriate for the truncated boundary based on the assumed traveling wave (i.e., Eq. (20a)):

$$
\frac{\partial p}{\partial x}-i k^{\prime} p=0
$$

Employing Eq. (22) in Eq. (9c), it yields:

$$
\mathbf{R}_{\mathbf{I}}^{e}=-\left(i k^{\prime}\right) \mathbf{L}_{\mathbf{I}}^{e} \mathbf{P}^{e},
$$

with the following definition:

$$
\mathbf{L}_{\mathrm{I}}^{e}=\frac{1}{\rho} \int_{\Gamma_{\mathrm{I}}^{e}} \mathbf{N} \mathbf{N}^{T} d \Gamma^{e} .
$$

Assembling $\mathbf{R}_{\mathrm{I}}^{e}$ for all fluid elements adjacent to truncation boundary leads to:

$$
\mathbf{R}_{\mathrm{I}}=-\left(\mathrm{i} k^{\prime}\right) \mathbf{L}_{\mathrm{I}} \mathbf{P} \text {. }
$$

This can now be substituted into Eq. (19) to obtain the FE equations of the coupled dam-reservoir system in its final form for the frequency domain as shown in Box I. It is also noticed that the lower matrix equation of (26) is multiplied by $\omega^{-2}$ in this process to obtain a symmetric dynamic stiffness matrix for the damreservoir system.

\subsection{Theoretical background pertinent to parameter $\boldsymbol{k}^{\prime}$}

The major remaining concept is the determination of parameter $k^{\prime}$. The relevant theoretical background will be discussed in this section. Of course, there are different available options which will be actually presented in the next section. However, prior to getting into a discussion on that, it is worthwhile to review some salient aspects of the exact analytical solution available for the domain $D$ (Figure 1) which is extremely helpful in this regard. It should be noted that this domain is actually eliminated from our problem.

$$
\left[\begin{array}{cc}
-\omega^{2} \mathbf{M}+(1+2 \beta i) \mathbf{K} & -\mathbf{B}^{T} \\
-\mathbf{B} & \omega^{-2}\left(-\omega^{2} \mathbf{G}+i k^{\prime} \mathbf{L}_{\mathbf{I}}+i \omega q \mathbf{L}_{\mathrm{II}}+\mathbf{H}\right)
\end{array}\right]\left\{\begin{array}{l}
\mathbf{r} \\
\mathbf{P}
\end{array}\right\}=\left\{\begin{array}{c}
-\mathbf{M} \mathbf{J} \mathbf{a}_{g}^{h} \\
\omega^{-2}\left(-\mathbf{B} \mathbf{J} \mathbf{a}_{g}^{h}\right)
\end{array}\right\}
$$


This is a regular semi-infinite region with constant depth $H$ extending to infinity in the upstream direction. The base of this region may be absorptive (i.e., $\alpha \neq 1$ ) or fully reflective (i.e., $\alpha=1$ or $q=0$ ). As mentioned, this was limited merely to the latter case in a previous study [19]. However, the more general case will be treated herein (i.e., no restriction on $\alpha$ ). Of course, it should be emphasized that we are still considering merely horizontal ground excitation similar to that study. Under these circumstances, the exact solution for this region may be written as follows [23]:

$$
\begin{gathered}
p(x, y, t)=\sum_{j=1}^{\infty} B_{j}\left[\cos \left(\lambda_{j} y\right)+\frac{i \omega q}{\lambda_{j}} \sin \left(\lambda_{j} y\right)\right] \\
e^{i\left(k_{j}^{\prime} x+\omega t\right)} .
\end{gathered}
$$

It is noted that the solution is composed of different modes, and amplitude $B_{j}$ depends on the existing conditions on the downstream face of that region. Parameters $\lambda_{j}$ are calculated through the following Eigenvalue problem:

$$
\cos \left(\lambda_{j} H\right)+\frac{i \omega q}{\lambda_{j}} \sin \left(\lambda_{j} H\right)=0 .
$$

Herein, $H$ represents the water depth. Moreover, parameters $\lambda_{j}$ and $k_{j}^{\prime}$ are related as follows:

$$
{k_{j}^{\prime}}^{2}+\lambda_{j}^{2}=\frac{\omega^{2}}{c^{2}}
$$

By employing Eq. (29), one would obtain:

$$
k_{j}^{\prime}= \pm i \sqrt{\lambda_{j}{ }^{2}-\frac{\omega^{2}}{c^{2}}} .
$$

Although there are two options in this definition, the negative sign is merely admissible for a semi-infinite region extending to infinity in the negative $x$-direction as in our present case. This is due to the fact that we are only interested in the modes which are decaying and propagating towards the upstream direction.

\subsubsection{Fully reflective reservoir base condition (i.e., special case)}

Let us now concentrate on the special case of the fully reflective base condition (i.e., $\alpha=1$ or $q=0$ ). Under these circumstances, Relations (27) and (28) are simplified as follows:

$$
\begin{aligned}
& p(x, y, t)=\sum_{j=1}^{\infty} B_{j}\left[\cos \left(\lambda_{j} y\right)\right] e^{i\left(k_{j}^{\prime} x+\omega t\right)}, \\
& \cos \left(\lambda_{j} H\right)=0 .
\end{aligned}
$$

Moreover, eigenvalues $\lambda_{j}$ are readily obtained:

$$
\lambda_{j}=\frac{(2 j-1) \pi}{2 H} .
$$

It is also noted that the $j$ th wavenumber $\left(k_{j}^{\prime}\right)$ becomes zero at a cut-off frequency referred to as the $j$ th natural frequency of the reservoir (i.e., $\omega_{j}^{r}$ ). This is obtained by substituting Eq. (33) into Eq. (30) under that condition which results in:

$$
\omega_{j}^{r}=\frac{(2 j-1) \pi c}{2 H} .
$$

Eq. (30) with the admissible negative sign may be also written as follows:

$$
k_{j}^{\prime}=\frac{\omega}{c}\left(\frac{-i(2 j-1)}{\Omega} \sqrt{1-\frac{\Omega^{2}}{(2 j-1)^{2}}}\right) .
$$

By means of dimensionless frequency $\Omega$, we have:

$$
\Omega=\frac{\omega}{\omega_{1}^{r}} .
$$

\subsection{Different options for defining parameter $k^{\prime}$}

Let us now describe some of the available options for selecting parameter $k^{\prime}$ in Eq. (26).

\section{Alternative 1}

As the first option, one presumes that the assumed planar wave is impinging on the truncation boundary perpendicularly. Thus, angle $\theta$ is zero, and $k^{\prime}$ is readily found from Eq. (21a):

$$
k^{\prime}=\frac{\omega}{c} \text {. }
$$

Accordingly, this may be envisaged as a limiting case for each $k_{j}^{\prime}$ (Eq. (35)) as $\Omega$ goes to infinity. Substituting Eq. (37) into Eq. (22) leads to what is known as the Sommerfeld boundary condition for the frequency and time domains, respectively [4]:

$$
\begin{aligned}
& \frac{\partial p}{\partial x}=\frac{i \omega}{c} p, \\
& \frac{\partial p}{\partial x}=\frac{1}{c} \frac{\partial p}{\partial t} .
\end{aligned}
$$

\section{Alternative 2}

The second option is to define $k^{\prime}$ based on an approximation of the first wavenumber assuming the full reflective base condition as a simplification. Thus, let us consider the first wavenumber for that special case (i.e., substituting $j=1$ into Eq. (35)):

$$
k_{1}^{\prime}=\frac{\omega}{c}\left(\frac{-i}{\Omega} \sqrt{1-\Omega^{2}}\right) .
$$

By employing the estimate $\sqrt{1-\Omega^{2}} \approx(1+i \Omega)$ on 
Eq. (39) and utilizing Eqs. (34) and (36), the following can be yielded:

$$
k^{\prime}=\frac{\omega}{c}-i \frac{\pi}{2 H} .
$$

Substituting Eq. (40) into Eq. (22) leads to what is known as Sharan boundary condition for the frequency and time domains, respectively [5]:

$$
\begin{aligned}
& \frac{\partial p}{\partial x}=\frac{i \omega}{c} p+\frac{\pi}{2 H} p, \\
& \frac{\partial p}{\partial x}=\frac{1}{c} \frac{\partial p}{\partial t}+\frac{\pi}{2 H} p .
\end{aligned}
$$

\section{Alternative 3}

The third option is what is proposed in this study. That is to define $k^{\prime}$ based on different wavenumbers for various frequency ranges. In particular, the following strategy is used:

$$
k^{\prime}=k_{1}^{\prime} ; \quad \text { initially as a default value. }
$$

$$
\begin{aligned}
& k^{\prime}=k_{j}^{\prime} \\
& \text { if }\left[\operatorname{Real}\left(\lambda_{j}^{2}-\frac{\omega^{2}}{c^{2}}\right) \leq 0 \text { and } \operatorname{Real}\left(\lambda_{j+1}^{2}-\frac{\omega^{2}}{c^{2}}\right)>0\right]
\end{aligned}
$$

for $j \geq 2$,

where $k_{j}^{\prime}$ is solved through Eq. (30) with a negative sign option. Of course, this requires the computation of $\lambda_{j}$ which is solved through the Eigenvalue problem Eq. (28) by the well-known Newton-Raphson approach.

It should be mentioned that for the special case of the full reflective base condition, the strategy may be equivalently written as follows:

$$
\begin{aligned}
& k^{\prime}=k_{1}^{\prime} ; \text { for }\left[0 \leq \Omega \leq 3 \text { or }\left(0 \leq \omega \leq \omega_{2}^{r}\right)\right] \\
& k^{\prime}=k_{j}^{\prime} ; \text { for }[(2 j-1) \leq \Omega \leq(2 j+1) \text { or } \\
& \left.\quad\left(\omega_{j}^{r}<\omega \leq \omega_{j+1}^{r}\right)\right] \text { and } j \geq 2,
\end{aligned}
$$

where $k_{j}^{\prime}$ is solved through Eq. (35), as proposed in the previous study [19]. It is also worthwhile to mention that, for the special case, $k^{\prime}$ would be either a real number or a pure imaginary number. While, in the general case, it could be a complex number having both real and imaginary components.

As mentioned above, in general, eigenvalues $\lambda_{j}$ are solved through Eq. (28) by utilizing the NewtonRaphson algorithm. This is carried out herein for the first five modes, and the results are depicted in Figure 2, similar to the work of Fenves and Chopra [24].
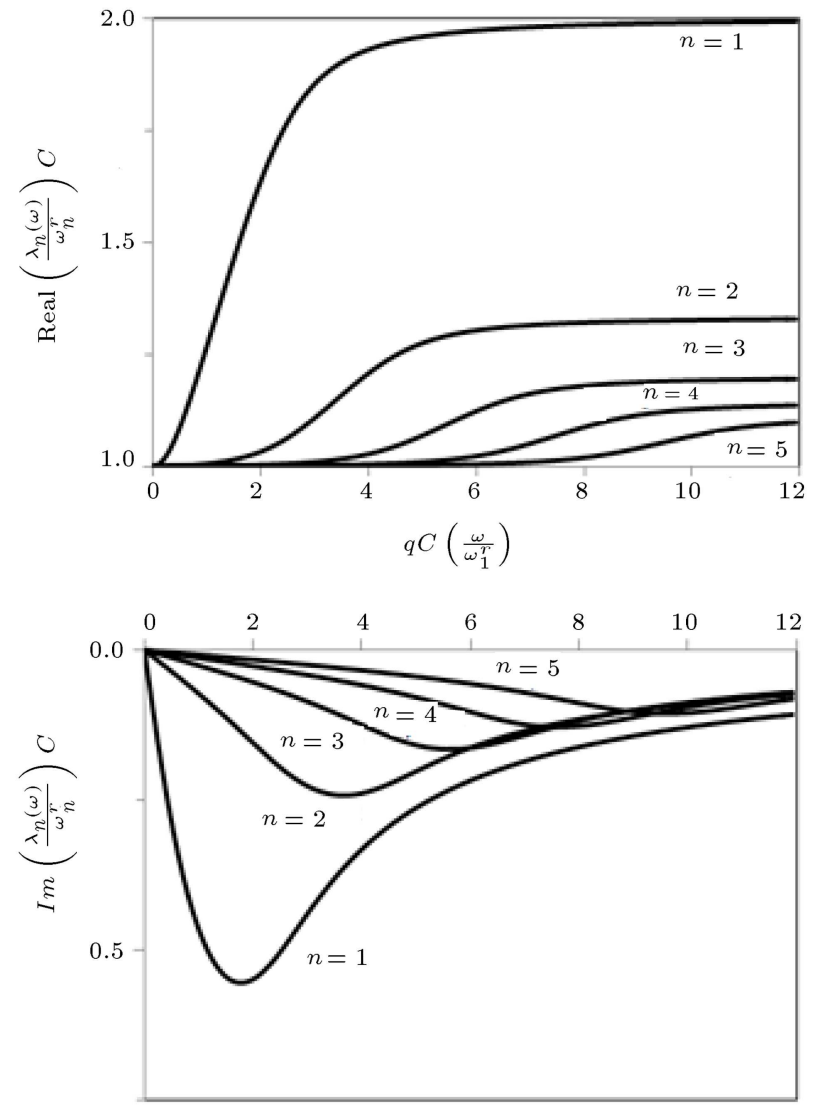

Figure 2. Variation of $\lambda_{j}$ with excitation frequency for the general case of reservoir base condition.

\section{Modelling and basic parameters}

The introduced methodology is employed to analyze an idealized dam-reservoir system. The details about modeling aspects, such as discretization, basic parameters, and the assumptions adopted, are summarized in this section.

\subsection{Models}

An idealized triangular dam with vertical upstream face and a downstream slope of 1:0.8 is considered on a rigid base. The dam is discretized by 20 isoparametric 8-node plane-solid finite elements.

As for the water domain, two strategies are adopted (Figure 3). For the FE-FE method of analysis which is our main procedure, only the near-field reservoir is discretized and the absorbing boundary condition is employed on the upstream truncation boundary according to different alternatives discussed. The length of this near-field region is denoted by $L$ and water depth is referred to as $H$. Three cases are considered. These are in particular the $L / H$ values of $0.2,1$, and 4 which represent low, medium, and high reservoir lengths. This region is discretized by 5, 25, and 100 isoparametric 8-node plane-fluid finite elements for the three above-mentioned $L / H$ values, respectively. 


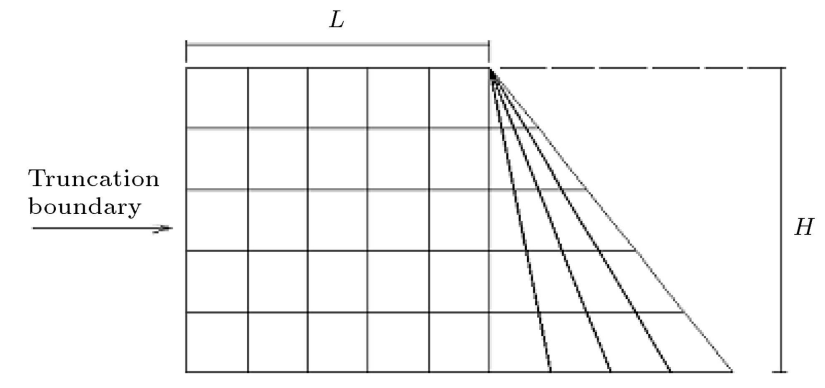

(a)

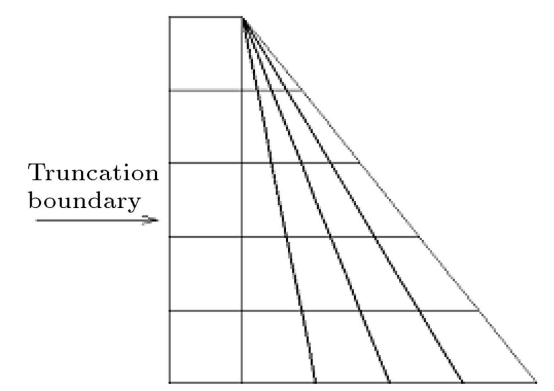

(b)

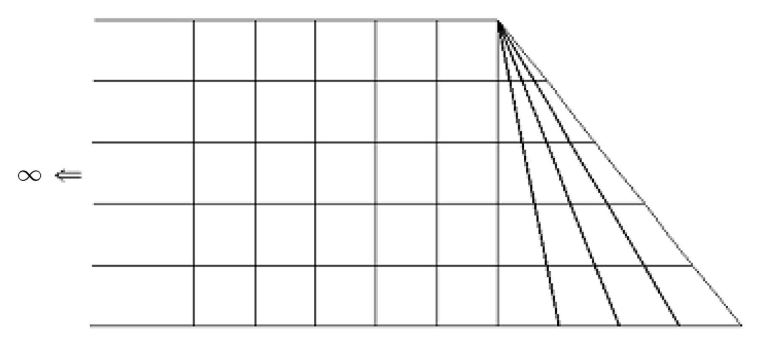

(c)

Figure 3. The dam-reservoir discretization for (a) FE-FE model $(L / H=1)$, (b) FE-FE model $(L / H=0.2)$, and (c) FE-(FE-HE) model $(L / H=1)$.

For the FE-(FE-HE) method of analysis, the reservoir domain is divided into two regions. The nearfield region is discretized by fluid finite elements, and the far-field region is treated by a fluid hyper-element. Of course, it should be emphasized that this option is merely utilized to obtain the exact solution [25]. Moreover, it is well known that the results are not sensitive in this case to the length of the near-field reservoir region or $L / H$ value.

\subsection{Basic parameters}

The dam body is assumed to be homogeneous and isotropic with linearly viscoelastic properties for mass concrete:

- Elastic modulus $\left(E_{d}\right)=27.5 \mathrm{GPa}$;

- Poison's ratio $=0.2$;

- Unit weight $=24.8 \mathrm{kN} / \mathrm{m}^{3}$;

- Hysteretic damping factor $\left(\beta_{d}\right)=0.05$;

- The impounded water is taken as inviscid and compressible fluid with a unit weight equal to 9.81
$\mathrm{kN} / \mathrm{m}^{3}$, and pressure wave velocity is $c=1440$ $\mathrm{m} / \mathrm{sec}$.

\section{Results}

It should be emphasized that all presented results herein are obtained by the FE-FE method discussed in different absorbing conditions applied on the truncation boundary. The only exception is for what is referred to as the exact response. That special case is carried out by the FE-(FE-HE) analysis technique.

The initial part of the study relates to a damreservoir system with a medium near-field reservoir length (i.e., $L / H=1$ (Figure $3(\mathrm{a}))$ ). This is examined for two different assumptions of fully reflective and absorptive reservoir base conditions (i.e., $\alpha=1$ and 0.75). For each model, three cases are considered. The only difference between these cases is a type of the absorbing boundary condition imposed on the truncation boundary. These are in particular based on alternatives I, II, and III (i.e., Sommerfeld, Sharan, and wavenumber conditions, respectively).

The transfer function for the horizontal acceleration at dam crest with respect to the horizontal ground acceleration is presented in Figure 4 for these three cases with the fully reflective reservoir base assumption $(\alpha=1)$. It is noted that the response in each case is plotted versus the dimensionless frequency. The normalization of excitation frequency is carried out with respect to $\omega_{1}$, which is defined as the natural frequency of the dam with an empty reservoir on the rigid foundation. Moreover, it is noticed that all cases are compared with the exact response.

It is observed that the response for the Sommerfeld condition case (i.e. the first case) has significant error near the fundamental frequency of the system, where the first major peak occurs. For the second case (Sharan B.C.), the error reduces at the first major peak (compared to the first case); however, it is observed that the response maintains a similar pattern. Moreover, error increases at the second major peak for this case, which shows the deficiency of the Sharan condition with respect to Sommerfeld B.C. for that frequency range.

The third case is related to the wavenumber approach. It is observed that the response agrees very well with the exact response, and the initial result of this technique reveals the promising behavior of this alternative.

Subsequently, similar plots are illustrated in Figure 5 for the same three alternatives with the absorptive reservoir base assumption $(\alpha=0.75)$.

It is observed that both Sommerfeld and Sharan cases still have noticeable errors near the fundamental frequency of the system, although it is reduced in comparison with the fully reflective cases mentioned 


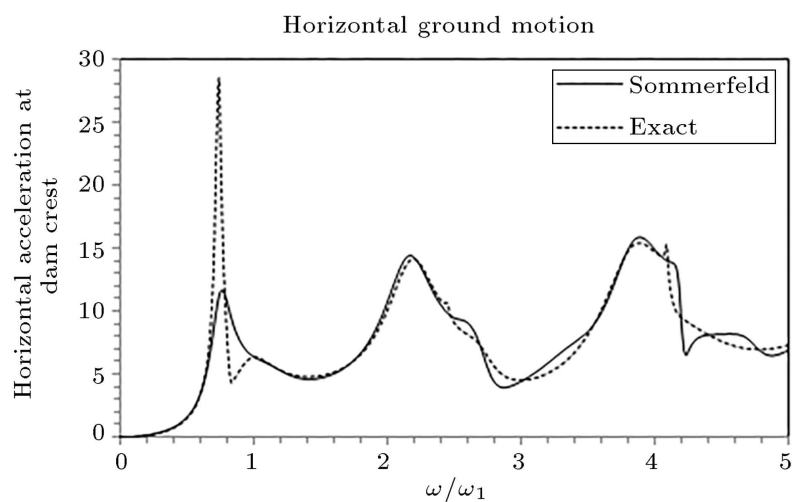

(a)

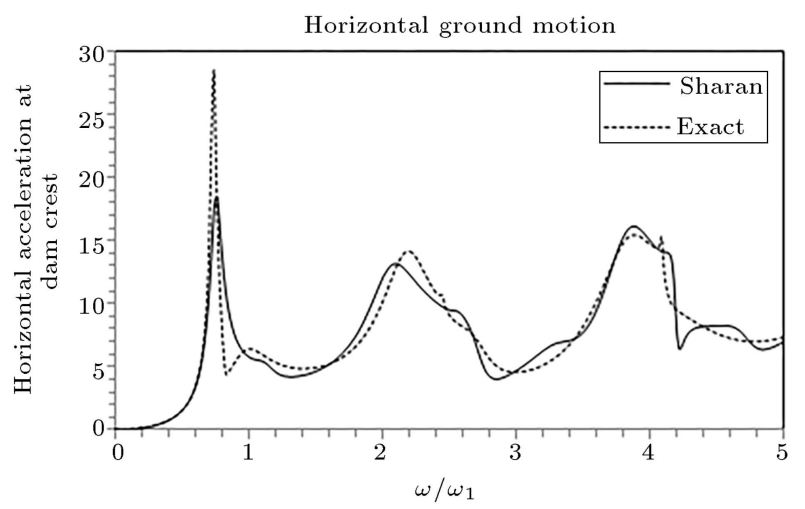

(b)

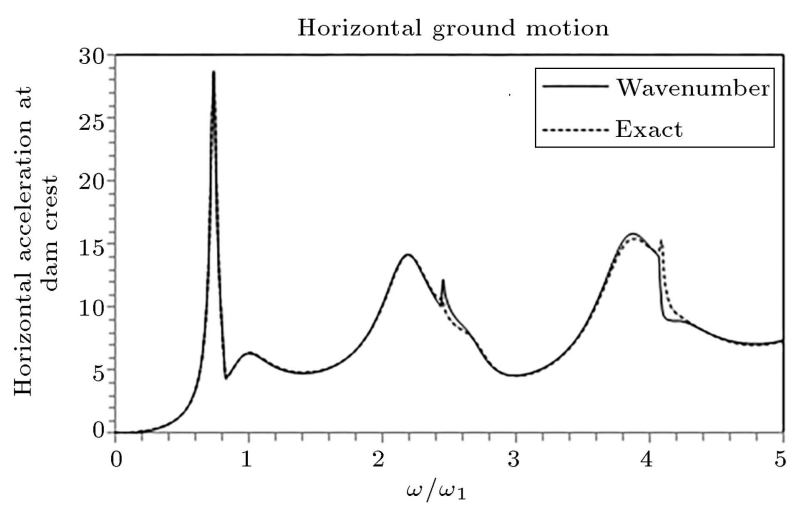

(c)

Figure 4. Horizontal acceleration at dam crest due to horizontal ground motion for the medium reservoir length and fully reflective reservoir base condition $(L / H=1$, $\alpha=1$ ) under different absorbing boundary condition alternatives: (a) Sommerfeld, (b) Sharan, and

(c) wavenumber.

above. For the wavenumber approach, the response is predicted very well, and the agreement with the exact result is almost perfect for this case.

In the second part of this study, it is decided to investigate the behavior of three alternatives I, II, and III (i.e., Sommerfeld, Sharan, and wavenumber approaches) for low near-field reservoir lengths. For this purpose, a very low reservoir length (i.e., $L / H=$ 0.2 (Figure $3(\mathrm{~b}))$ ) is considered that is a challenging

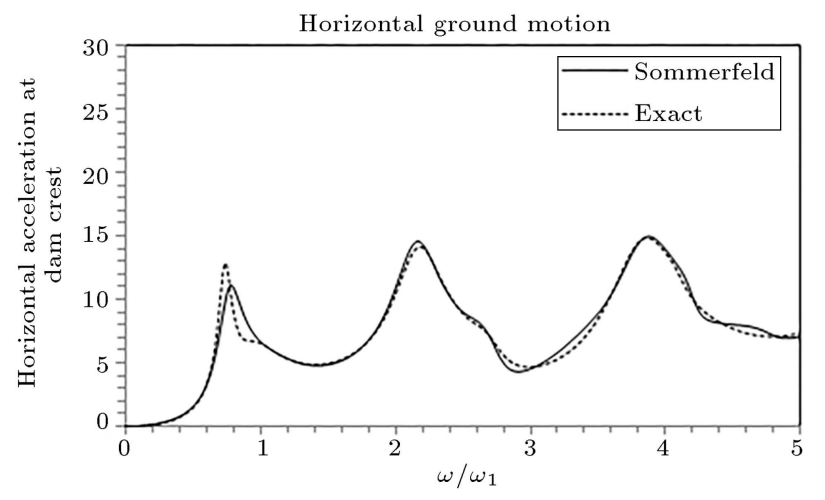

(a)



(b)

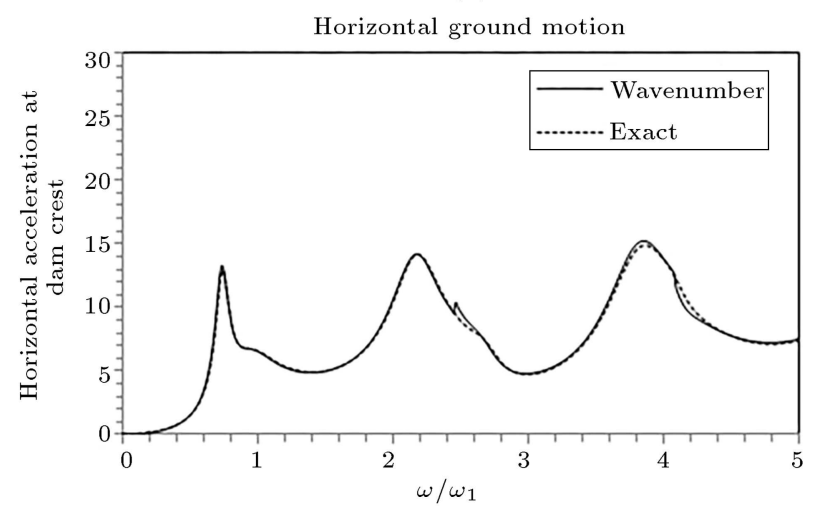

(c)

Figure 5. Horizontal acceleration at dam crest due to the horizontal ground motion for the medium reservoir length and absorptive reservoir base condition $(L / H=1$,

$\alpha=0.75)$ under different absorbing boundary condition alternatives: (a) Sommerfeld, (b) Sharan, and (c) wavenumber.

test for examining any type of the absorbing boundary condition.

Similar to the medium reservoir length, this is examined for two different assumptions of fully reflective and absorptive reservoir base conditions (i.e., $\alpha=1$ and 0.75 ).

The responses for the fully reflective reservoir base condition $(\alpha=1)$ are presented in Figure 6 . It is observed that there are significant errors in the responses for the Sommerfeld and Sharan condition cases. 


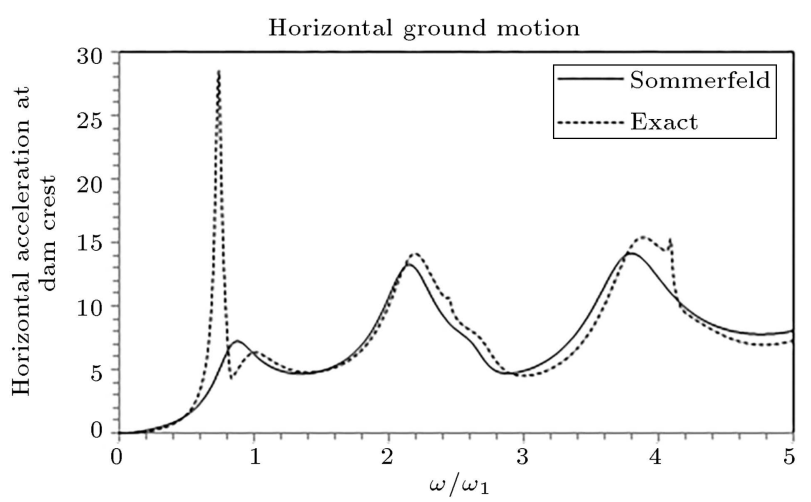

(a)

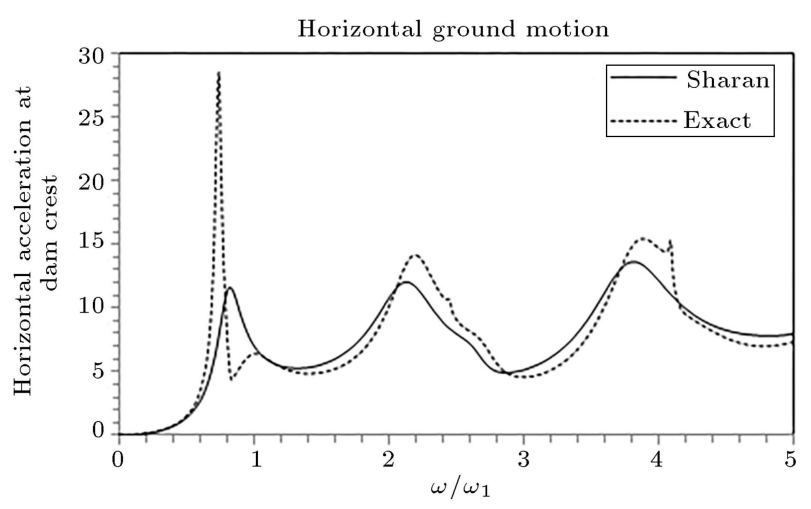

(b)

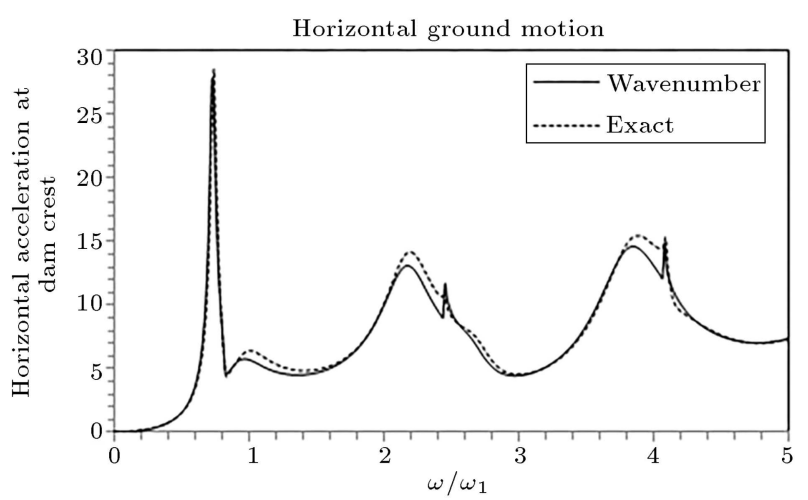

(c)

Figure 6. Horizontal acceleration at dam crest due to the horizontal ground motion for the low reservoir length and fully reflective reservoir base condition $(L / H=0.2, \alpha=1)$ under different absorbing boundary condition alternatives: (a) Sommerfeld, (b) Sharan, and (c) wavenumber.

Moreover, the errors have increased in comparison with the medium reservoir length results (Figure 6 versus Figure 4). For the wavenumber approach, the response is still close to the exact response in most frequency ranges. However, there exist errors in the range of $5 \%$ at the major peaks of the response. This is still believed to be a remarkable result for such a challenging test.

Similar plots are illustrated in Figure 7 for the same three alternatives with the absorptive reservoir base assumption $(\alpha=0.75)$. It is observed that



(a)



(b)

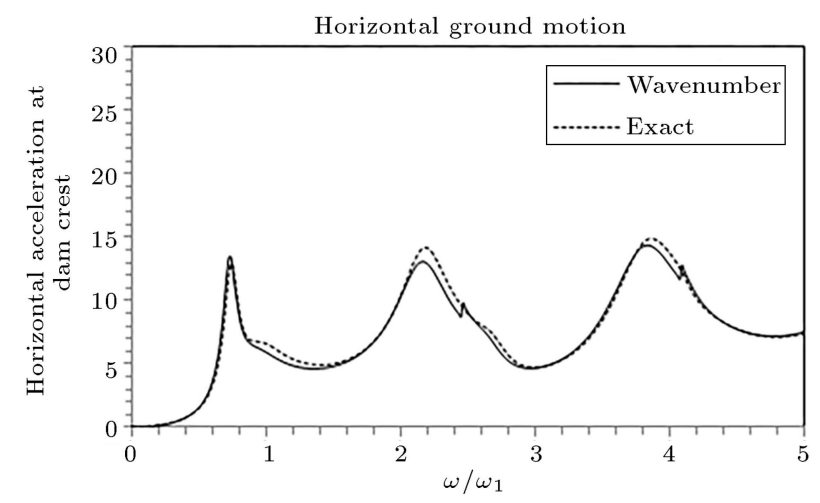

(c)

Figure 7. Horizontal acceleration at dam crest due to the horizontal ground motion for the low reservoir length and absorptive reservoir base condition $(L / H=0.2, \alpha=0.75)$ under different absorbing boundary condition alternatives: (a) Sommerfeld, (b) Sharan, and (c) wavenumber.

there are still significant errors for the Sommerfeld and Sharan condition cases. However, as expected, the errors have decreased with respect to the fully reflective reservoir condition (Figure 7 versus Figure 6). For the wavenumber approach, it is observed that the error at the first major peak of the response diminishes with respect to the fully reflective reservoir base condition (Figure 7 versus Figure 6), and the response agrees relatively well with the exact response for the whole frequency range. 
In the last part of this study, it is worthwhile to investigate the behavior of three alternatives I, II, and III (i.e., Sommerfeld, Sharan, and wavenumber approaches) for high near-field reservoir lengths. For this purpose, a relatively high reservoir length (i.e., $L / H=4)$ is considered. Similar to the previous reservoir lengths, this is examined for two different assumptions of fully reflective and absorptive reservoir base conditions (i.e., $\alpha=1$ and 0.75 ).

The responses for the fully reflective reservoir base condition $(\alpha=1)$ are presented in Figure 8 . It is noticed that responses for the sommerfeld and Sharan condition cases have improved greatly in comparison with the low or even medium length results discussed previously (Figure 8 versus Figure 6 or 4 ). However, some kinds of noise or distortion are noticed in the response of both these cases, especially for higher frequencies. As for the wavenumber approach, it is noticed that the response agrees very well with the exact response, similar to the behavior noticed for the medium reservoir length. Moreover, there are few signs of distortions in the response for the high reservoir length, contrary to what was noticed for the other two well-known alternatives I and II (the Sommerfeld and Sharan condition cases).

Similar plots are also illustrated in Figure 9 for the same three alternatives with the absorptive reservoir base assumption $(\alpha=0.75)$. It is observed that all three alternatives reveal good behavior, and very close agreement is obtained with respect to the exact response for the whole frequency range under these circumstances (i.e., high reservoir length and absorptive reservoir base assumption $(\alpha=0.75)$ ).

Overall, it can be concluded that the maximum error of the wavenumber approach is in the range of $5 \%$ at the major peaks of the response. This occurs only for the very low reservoir lengths and fully reflective reservoir base condition. This is a remarkable result for any kind of robust truncation boundary simulation that one may expect. It is also worthwhile to mention that, in general, the fundamental frequency of the system is not captured correctly when both Sommerfeld and Sharan B.C. are employed unless the reservoir length is selected as a high value. This is true especially for cases in which low reservoir lengths are utilized in the model (Figures 6 and 7 ). However, the fundamental frequency of the system is captured correctly for the wavenumber approach, even in cases of the low reservoir length (Figures 6(c) and $7(\mathrm{c})$ ).

\section{Conclusions}

The formulation based on the FE-FE procedure for dynamic analysis of concrete dam-reservoir systems was reviewed. Moreover, several options were discussed for imposing a local type of absorbing condition on the

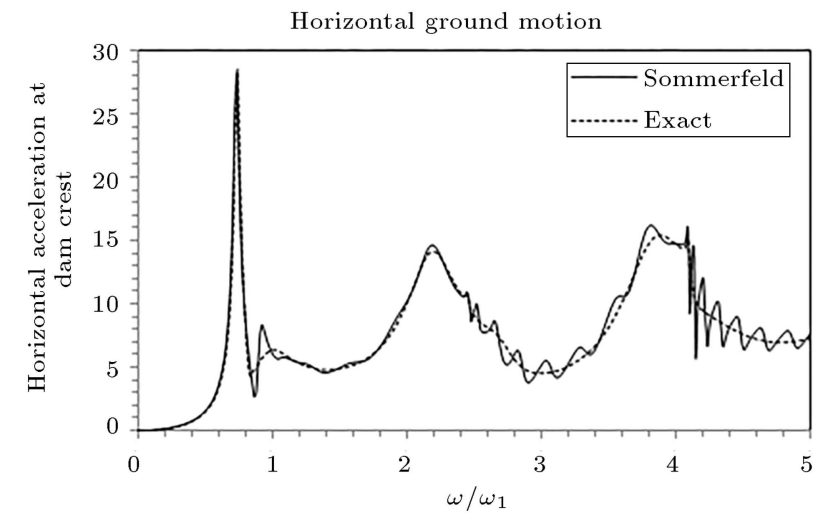

(a)

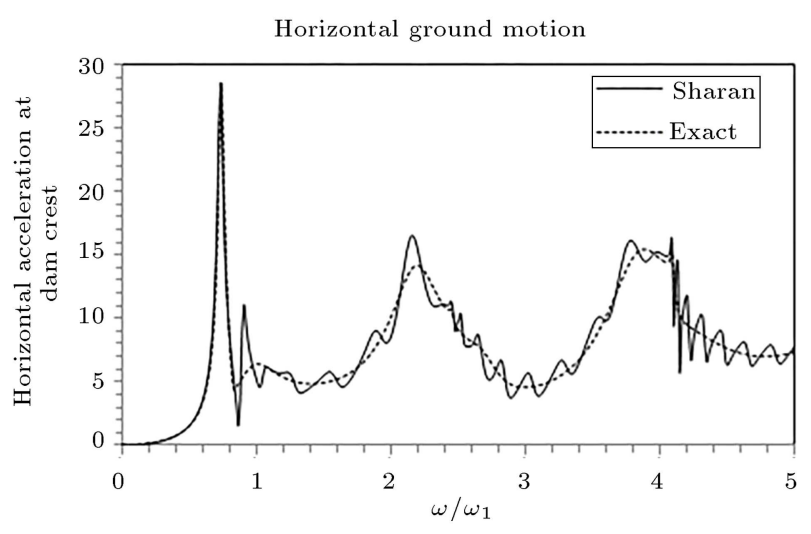

(b)

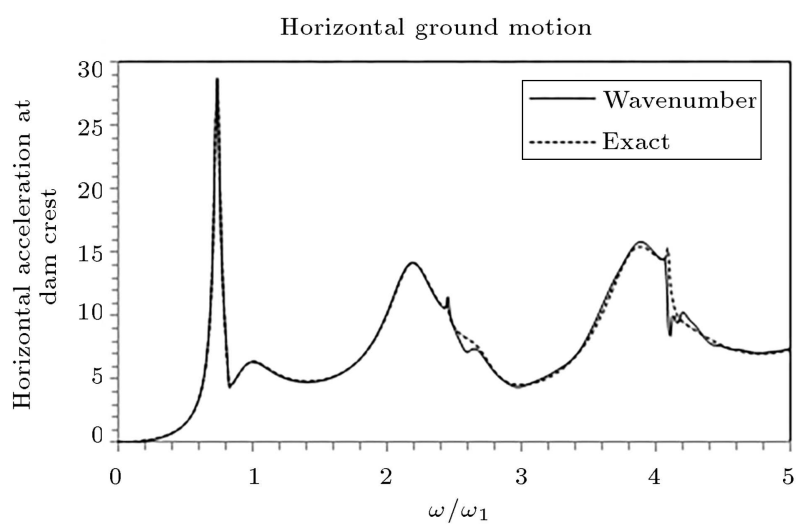

(c)

Figure 8. Horizontal acceleration at dam crest due to the horizontal ground motion for the high reservoir length and fully reflective reservoir base condition $(L / H=4, \alpha=1)$ under different absorbing boundary condition alternatives: (a) Sommerfeld, (b) Sharan, and (c) wavenumber.

truncation boundary of the water domain. A specialpurpose finite-element program was enhanced for this investigation. Thereafter, the response of an idealized triangular dam was studied due to the horizontal ground motion for different alternatives employed as an absorbing boundary condition. The main approach which was emphasized and proposed in this study is referred to as the wavenumber approach. 


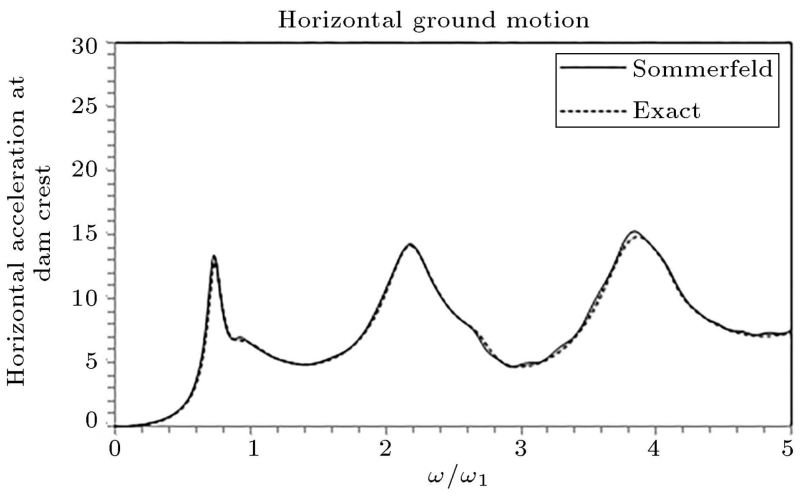

(a)

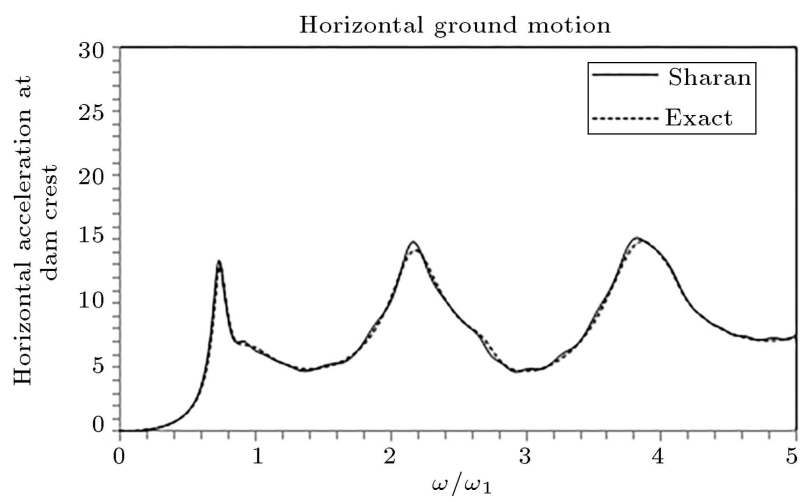

(b)

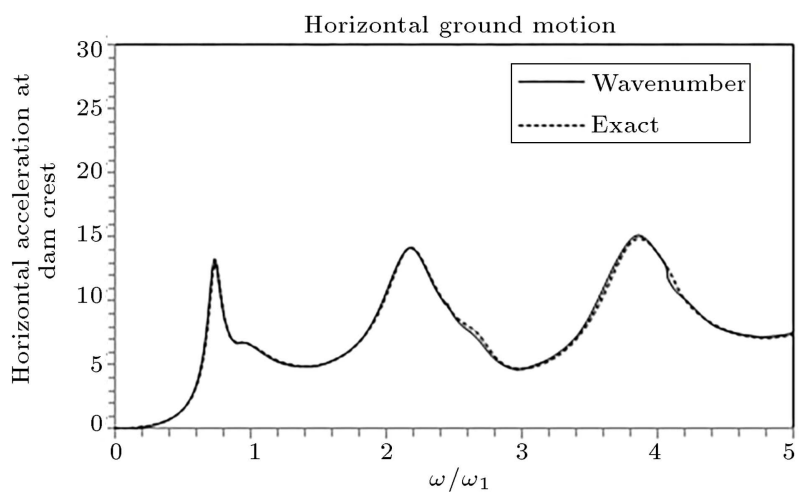

(c)

Figure 9. Horizontal acceleration at dam crest due to the horizontal ground motion for the high reservoir length and absorptive reservoir base condition $(L / H=4, \alpha=0.75)$ under different absorbing boundary condition alternatives: (a) Sommerfeld, (b) Sharan, and (c) wavenumber.

Overall, the main conclusions obtained by the present study can be listed as follows.

With regard to the Sommerfeld and Sharan absorbing conditions:

- In general, the fundamental frequency of the system is not captured correctly for both of these approaches unless the reservoir length is selected as a high value. This is especially true for cases in which low reservoir lengths are utilized in the model;
- There are significant errors occurring on the response at the fundamental frequency of the system for low or even medium reservoir lengths. The error decreases for the absorptive reservoir base condition, or as the reservoir length increases;

- Obviously, the main advantage of these two conditions is that both of them can be readily utilized in the time and frequency domains.

With regard to the wavenumber absorbing condition:

- The fundamental frequency of the system is captured correctly for the wavenumber approach, even in cases of a low reservoir length;

- It is concluded that the maximum error for the wavenumber approach is in the range of $5 \%$ at the major peaks of the response. This occurs only for the very low reservoir lengths and fully reflective reservoir base condition. This is a remarkable result for any kind of robust truncation boundary simulation that one may expect;

- Obviously, the main disadvantage of this condition is that it cannot be utilized in time domain, and it is only suitable for frequency domain;

- The wavenumber approach is ideal from the programming point of view due to the local nature of wavenumber condition imposed on truncation boundary. It can also be deemed as a great substitute for the rigorous FE-(FE-HE) type of analysis, which is heavily dependent on a hyperelement as its main core. It is undeniable that the rigorous approach is significantly more complicated from the programming point of view and much more computationally expensive.

\section{References}

1. Hall, J.F. and Chopra, A.K. "Two-dimensional dynamic analysis of concrete gravity and embankment dams including hydrodynamic effects", Earthq. Eng. Struct. D., 10(2), pp. 305-332 (1982).

2. Waas, G. "Linear two-dimensional analysis of soil dynamics problems in semi-infinite layered media", Ph.D. Dissertation, University of California, Berkeley, California (1972).

3. Khazaee, A. and Lotfi, V. "Application of perfectly matched layers in the transient analysis of damreservoir systems", Soil Dynamics and Earthquake Engineering, 60(1), pp. 51-68 (2014).

4. Sommerfeld, A., Partial Differential Equations in Physics, Academic Press, NY (1949).

5. Sharan, S.K. "Time domain analysis of infinite fluid vibration", Int. J. Numer. Meth. Eng., 24(5), pp. 945958 (1987). 
6. Berenger, J.P. "A perfectly matched layer for the absorption of electromagnetic waves", J. Comput. Phys., 114(2), pp. 185-200 (1994).

7. Chew, W.C. and Weedon, W.H. "A 3D perfectly matched medium from modified Maxwell's equations with stretched coordinates", Microw. Opt. Techn. Let., 7(13), pp. 599-604 (1994).

8. Basu, U. and Chopra, A.K. "Perfectly matched layers for time-harmonic elastodynamics of unbounded domains: theory and finite-element implementation", Comput. Meth. Appl. Mech. Eng., 192(11-12), pp. 1337-1375 (2003).

9. Jiong, L., Jian-wei, M., and Hui-zhu, Y. "The study of perfectly matched layer absorbing boundaries for $\mathrm{SH}$ wave fields", Appl. Geophys., 6(3), pp. 267-274 (2009).

10. Zhen, Q., Minghui, L., Xiaodong, Z., Yao, Y., Cai, Z., and Jianyong, S. "The implementation of an improved NPML absorbing boundary condition in elastic wave modeling", Appl. Geophys., 6(2), pp. 113-121 (2009).

11. Kim, S. and Pasciak, J.E. "Analysis of cartesian PML approximation to acoustic scattering problems in R2", Wave Motion, 49, pp. 238-257 (2012).

12. Higdon, R.L. "Absorbing boundary conditions for difference approximations to the multi-dimensional wave equation", Math. Comput., 47(176), pp. 437-459 (1986).

13. Givoli, D. and Neta, B. "High order non-reflecting boundary scheme for time-dependent waves", J. Comput. Phys., 186(1), pp. 24-46 (2003).

14. Hagstrom, T. and Warburton, T. "A new auxiliary variable formulation of high order local radiation boundary condition: corner compatibility conditions and extensions to first-order systems", Wave Motion, 39(4), pp. 327-338 (2004).

15. Givoli, D., Hagstrom, T., and Patlashenko, I. "Finiteelement formulation with high-order absorbing conditions for time-dependent waves", Comput. Meth. Appl. M., 195(29-32), pp. 3666-3690 (2006).

16. Hagstrom, T., Mar-Or, A., and Givoli, D. "Highorder local absorbing conditions for the wave equation: extensions and improvements", J. Comput. Phys., 227, pp. 3322-3357 (2008).

17. Rabinovich, D., Givoli, D., Bielak, J., and Hagstrom, T. "A finite element scheme with a high order absorbing boundary condition for elastodynamics", Comput. Meth. Appl. Mech., 200, pp. 2048-2066 (2011).
18. Samii, A. and Lotfi, V. "High-order adjustable boundary condition for absorbing evanescent modes of waveguides and its application in coupled fluid-structure analysis", Wave Motion, 49(2), pp. 238-257 (2012).

19. Lotfi, V. and Samii, A. "Dynamic analysis of concrete gravity dam-reservoir systems by wavenuber approach in the frequency domain", Earthquakes and Structures, 3(3-4), pp. 533-548 (2012).

20. Lotfi, V. and Samii, A. "Frequency domain analysis of concrete gravity dam-reservoir systems by wavenumber approach" , Proc. 15th World Conference on Earthquake Engineering, Lisbon, Portugal (2012a).

21. Zienkiewicz, O.C., Taylor, R.L., and Zhu, J.Z., The Finite Element Method, Butterworth-Heinemann (2013).

22. Chopra, A.K. "Hydrodynamic pressure on dams during earthquake", J. Eng. Mech.-ASCE, 93, pp. 205-223 (1967).

23. Chopra, A.K., Chakrabarti, P., and Gupta, S. "Earthquake response of concrete gravity dams including hydrodynamic and foundation interaction effects", Report No. EERC-80/01, University of California, Berkeley (1980).

24. Fenves, G. and Chopra, A.K. "Effects of reservoir bottom absorption and dam-water-foundation interaction on frequency response functions for concrete gravity dams", Earthq. Eng. Struct. D., 13, pp. 13-31 (1985).

25. Lotfi, V. "Frequency domain analysis of gravity dams including hydrodynamic effects", Dam Engineering, 12(1), pp. 33-53 (2001).

\section{Biographies}

Mehran Jafari received his MS degree in Civil Engineering from the Amirkabir University of Technology (Tehran Polytechnic), Tehran, Iran in 2014, where he also received his BS degree in 2011, respectively. His research interests include fluid-structure interaction, finite element, and structural analysis.

Vahid Lotfi was born on 1960 in Tehran, Iran. $\mathrm{He}$ received his $\mathrm{BS}, \mathrm{MS}$, and $\mathrm{PhD}$ degrees in Civil Engineering from the University of Texas at Austin, USA. He joined Amirkabir University of Technology, Tehran in 1986, and has been a full professor at that university since 2005. His research interests include finite element, fluid-structure interaction, concrete dams and earthquake engineering. 